\title{
Histerosalpingografía virtual por TC multidetector de 64 filas. Hallazgos en 2.500 pacientes
}

\author{
Drs. Patricia Carrascosa(1), Carlos Capuñay(1), Mariano Baronio(2), Javier Vallejos(1), Sergio Papier(2), \\ Jorge Carrascosa ${ }^{(1)}$.
}

1. Diagnóstico Maipú, Buenos Aires, Argentina.

2. CEGYR, Ciudad Autónoma de Buenos Aires, Argentina.

\section{4-Row multidetector CT virtual hysterosalpingography. Findings in 2500 patients}

\begin{abstract}
Objective. To depict typical features of virtual hysterosalpingography (VHSG) in infertile patients. Material and methods: 2500 VHSG performed using a 64-row MDCT were evaluated. Results: Cervical abnormalities visualized corresponded to parietal irregularities (21\%); thickened folds (9\%); polyps (11\%); diverticula (5\%); cervical strictures (8\%), and synechiae (1.5\%). At the level of uterine cavity, polyps (44\%); submucous myomas (8\%); intramural and subserosal fibroids (11\%); synechiae (10\%); malformations (10\%); adenomyosis $(4 \%)$, and cesarean section scar (3\%). Unilateral hydrosalpinx (7\%) and bilateral hydrosalpinx (2\%). Tubal obstruction was reported in $6 \%$ of cases. The average radiation dose was $0.94 \mathrm{mSv}$. Eighty-four percent of the patients reported mild pain or no postoperative discomfort. Conclusions: The virtual hysterosalpingography allowed a proper assessment of the internal genital organs, providing useful diagnostic information on infertility and other gynecological disorders. It constitutes a virtually painless, low-dose radiation technique, besides being well tolerated by patients.
\end{abstract}

Keywords: Fallopian tubes, Multidetector computed tomography, Uterus, Virtual hysterosalpingography, Virtual studies.

Resumen: Objetivo. Ilustrar los hallazgos característicos de la histerosalpingografía virtual (HSG-V) en pacientes en estudio de infertilidad. Material y métodos: Se evaluaron 2500 HSG-V efectuadas con un equipo de TCMD de 64 filas. Resultados A nivel del cuello se visualizaron irregularidades parietales (21\%); pliegues engrosados (9\%); pólipos (11\%); divertículos (5\%); estrechez (8\%; y sinequias (1,5\%). A nivel de la cavidad: pólipos (44\%); miomas submucosos (8\%); intramurales y subserosos (11\%); y sinequias (10\%); malformaciones (10\%); adenomiosis (4\%); cicatriz de cesárea (3\%). Hidrosalpinx unilateral (7\%) y bilateral (2\%); obstrucción tubaria en el $6 \%$ de los casos. La dosis de radiación promedio fue $0.94 \mathrm{mSv}$. El $84 \%$ de los pacientes refirieron disconfort leve o ausente. Conclusiones: La HSG-Virtual permitió una adecuada evaluación de los órganos genitales internos, brindando información diagnóstica útil en infertilidad y otros desórdenes ginecológicos. Es una técnica poco dolorosa, bien tolerada por las pacientes y con baja dosis de radiación.

Palabras clave: Estudios virtuales, Histerosalpingografía virtual, Tomografía computada multidetector, Trompas uterinas, Útero.

Carrascosa P y cols. Histerosalpingografía virtual por TC multidetector de 64 filas. Hallazgos en 2.500 pacientes. Rev Chil Radiol 2011; 17(3): 113-117.

Correspondencia: Dra. Patricia Carrascosa / investigacion@diagnosticomaipu.com.ar

Trabajo recibido el 21 de julio de 2011, aceptado para publicación el 16 de agosto de 2011.

\section{Introducción}

La histerosalpingografía virtual (HSG-V) es un nuevo método mínimamente invasivo para el diagnóstico del sistema reproductor femenino. Se realiza con tomógrafos multidetector (TCMD), los mejores resultados obtenidos con la generación de 64 filas de detectores $^{(1,2)}$. La mayor utilidad del método radica en la evaluación de la esterilidad primaria y secundaria(3,4).

A lo largo de más de 6 años de experiencia, nuestro grupo de trabajo ha evaluado y desarrollado las aplicaciones clínicas de la HSG-V, con resultados superiores a los arrojados por la histerosalpingografía convencional (HSG), tanto en la evaluación de la patología uterina como de las trompas ${ }^{(3-5)}$. 
El propósito de este trabajo es ilustrar diferentes aspectos de la HSG-V, ilustrando los hallazgos típicos y su diagnóstico diferencial con otras patologías en pacientes en estudio de infertilidad.

\section{Material y métodos Pacientes}

Se evaluaron en forma retrospectiva 2.500 pacientes de sexo femenino (edad promedio $36.2 \pm 3.6$ años) derivados a nuestra institución para realizar un estudio de HSG-V. Todos los estudios se realizaron con un tomógrafo de 64 filas. El protocolo de trabajo fue aprobado por el comité de ética institucional. El médico radiólogo actuante completó un formulario de historia clínica de la paciente. Todos los estudios se realizaron en la fase folicular del ciclo menstrual. Los criterios de exclusión incluyeron: presencia de embarazo, infección pélvica activa o aquellos casos en que la paciente se encontrara en una fecha posterior al día 11 del ciclo menstrual habiendo mantenido relaciones sexuales los días previos.

\section{Descripción del procedimiento}

Los exámenes se efectuaron con un equipo de 64 filas de detectores (Brilliance 64; Philips Medical Systems, Highland Heights, $\mathrm{OH}$ ). Los parámetros técnicos utilizados fueron: cortes de $0.9 \mathrm{~mm}$ de espesor; $0.45 \mathrm{~mm}$ de intervalo de reconstrucción; 120 $\mathrm{KV}$; $100-150 \mathrm{mAs}$; tiempo de rotación de tubo de 0.5 segundos. A todas las pacientes se les indicó retención urinaria de 2 horas. Los procedimientos se realizaron con la paciente en posición supina sobre la mesa del tomógrafo. Se utilizó una solución de yodo-povidona y gasas estériles para la antisepsia del periné y luego de la colocación de un espéculo estéril en la vagina, para la antisepsia del cuello uterino.

Sin realizar clampeo cervical ni fijación de otro tipo, se colocó una fina cánula plástica a nivel del orificio cervical externo, por la cual se instiló en la cavidad uterina un volumen total de 10 a $20 \mathrm{ml}$ de una dilución de contraste yodado [ $3 \mathrm{ml}$ de contraste hidrosoluble (iobitridol; Xenetix 350, Laboratorios Temis Lostaló, Buenos Aires, Argentina) y $17 \mathrm{ml}$ de solución salina].

Procesamiento de las imágenes

1) Imágenes axiales

2) Reconstrucciones multiplanares (MPR)

3) Máxima intensidad de proyección (MIP)

4) Volume rendering

5) Endoscopía virtual

Crea imágenes del interior del canal cervical y cavidad uterina, similares a las obtenidas por histeroscopía, permitiendo una correcta delineación de las lesiones endocavitarias. En casos de hidrosalpinx, permite también ver el interior de la trompa uterina.

\section{Hallazgos}

Los exámenes de HSG-V fueron divididos en estudios con hallazgos normales y patológicos. Las patologías se clasificaron de acuerdo a su localización (cervical, uterina, tubaria).

\section{Resultados}

A nivel del cuello se visualizaron irregularidades parietales $(21 \%)$, engrosamiento de pliegues $(9 \%)$, pólipos (11\%), divertículos (5\%), estrechez (8\%), y sinequias $(1,5 \%)$.

A nivel de la cavidad uterina se evidenciaron pólipos (44\%), miomas submucosos (8\%) y sinequias (10\%); miomas intramurales y subserosos (11\%), malformaciones (10\%), adenomiosis $(4 \%)$ y cicatriz de cesárea (3\%).

Se identificó hidrosalpinx unilateral (7\%) y bilateral (2\%); obstrucción tubaria en el $6 \%$ de los casos.

El tiempo promedio fue de $3.2 \pm 1.0 \mathrm{seg}$. La dosis de radiación promedio fue de $0.94+/-0.1 \mathrm{mSv}$. El $84 \%$ de los pacientes refirieron discomfort leve o ausente.

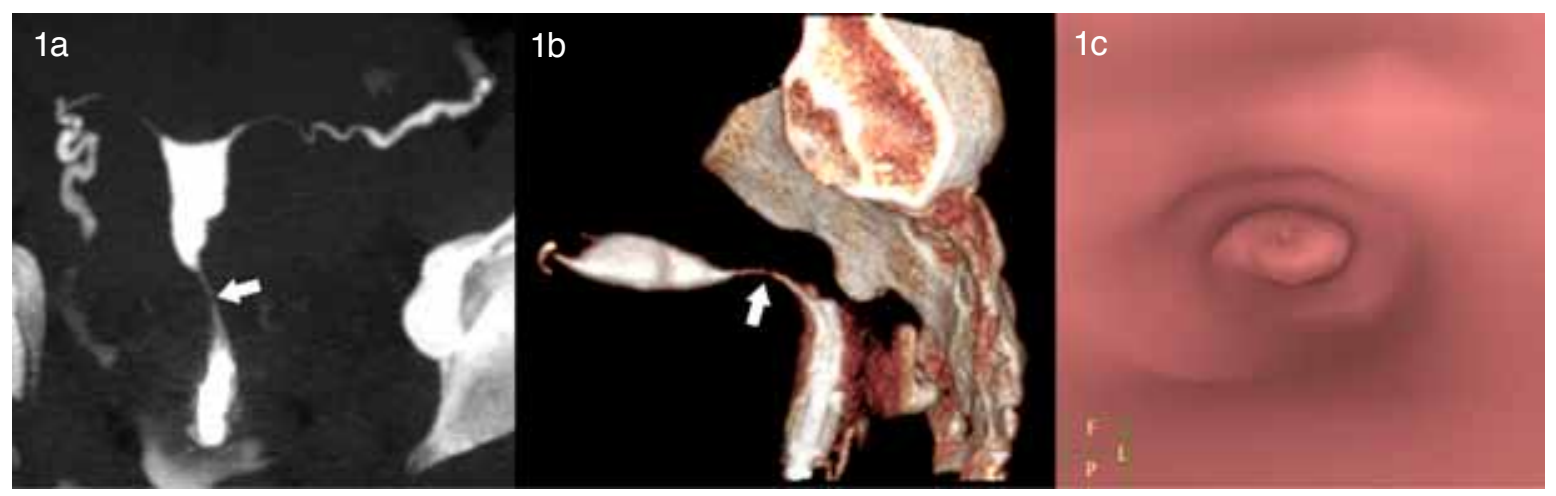

Figura 1. Estenosis difusa del canal cervical (flecha). A. Reconstrucción coronal en máxima intensidad de proyección. B. Reconstrucción sagital 3D volumen rendering. C. Endoscopía virtual. 


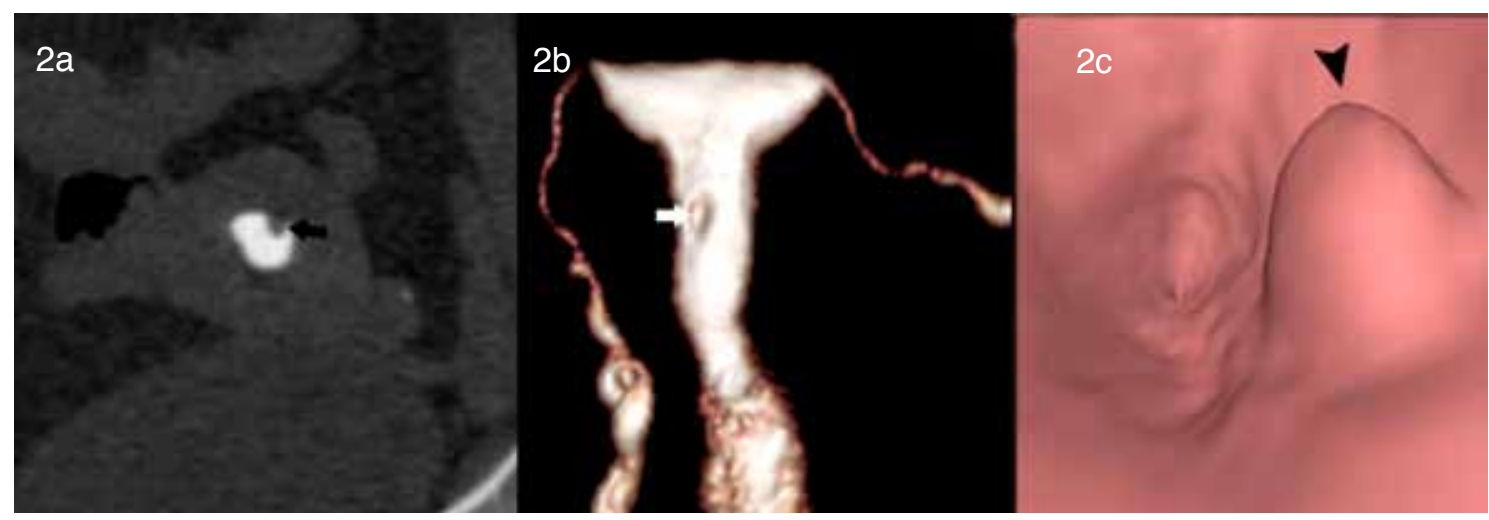

Figura 2. Pólipo endometrial (flecha). A. Imagen axial que muestra una lesión sobre-elevada sobre la pared anterior de la cavidad uterina. B. Reconstrucción coronal 3D volumen rendering. C. Endoscopía virtual.

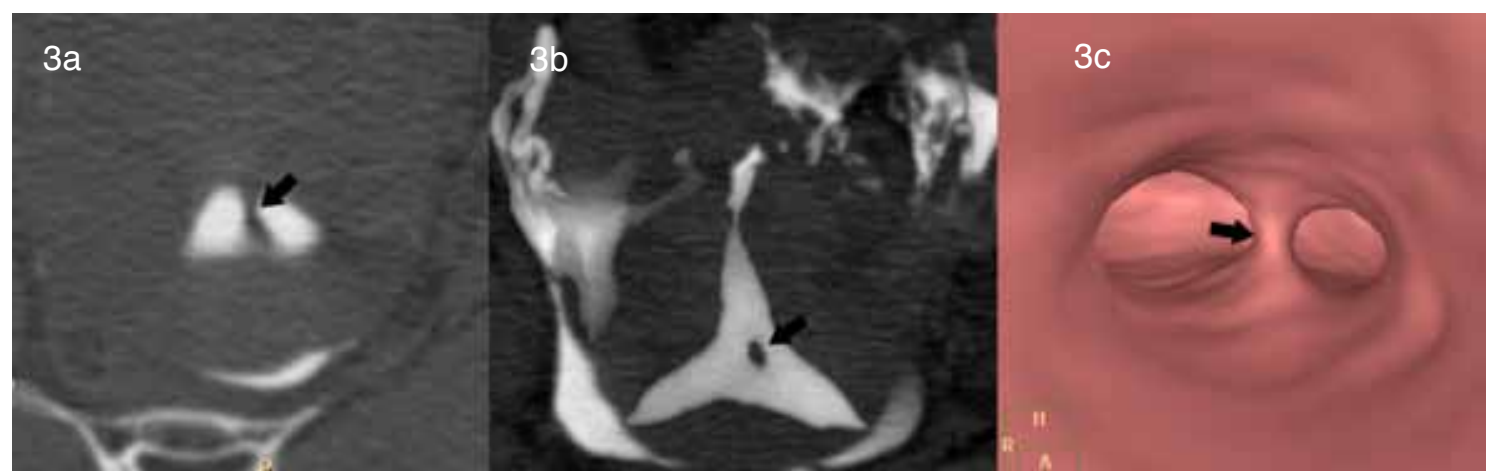

Figura 3. Sinequia uterina (flecha). A. Imagen axial que muestra un defecto de relleno lineal grueso a nivel de la cavidad uterina. B. Reconstrucción coronal en máxima intensidad de proyección. C. Endoscopía virtual.

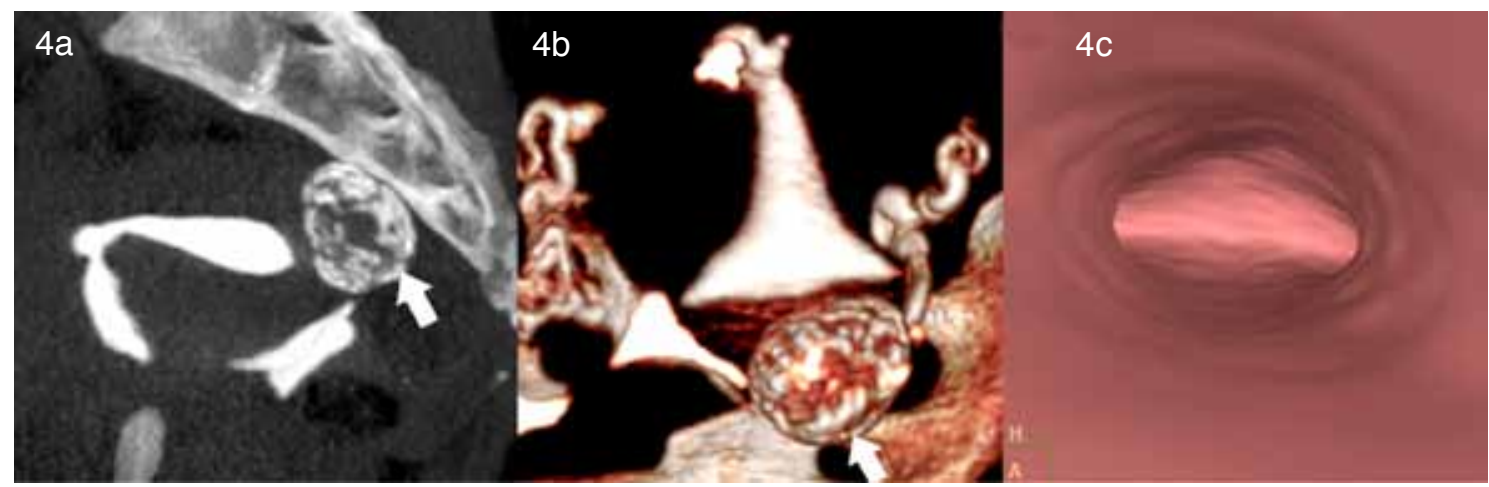

Figura 4. Mioma intramural fúndico calcificado (flecha). A. Reconstrucción sagital en máxima intensidad de proyección. $B$. Reconstrucción sagital 3D volumen rendering. $C$. Endoscopía virtual. La cavidad uterina no muestra alteraciones.

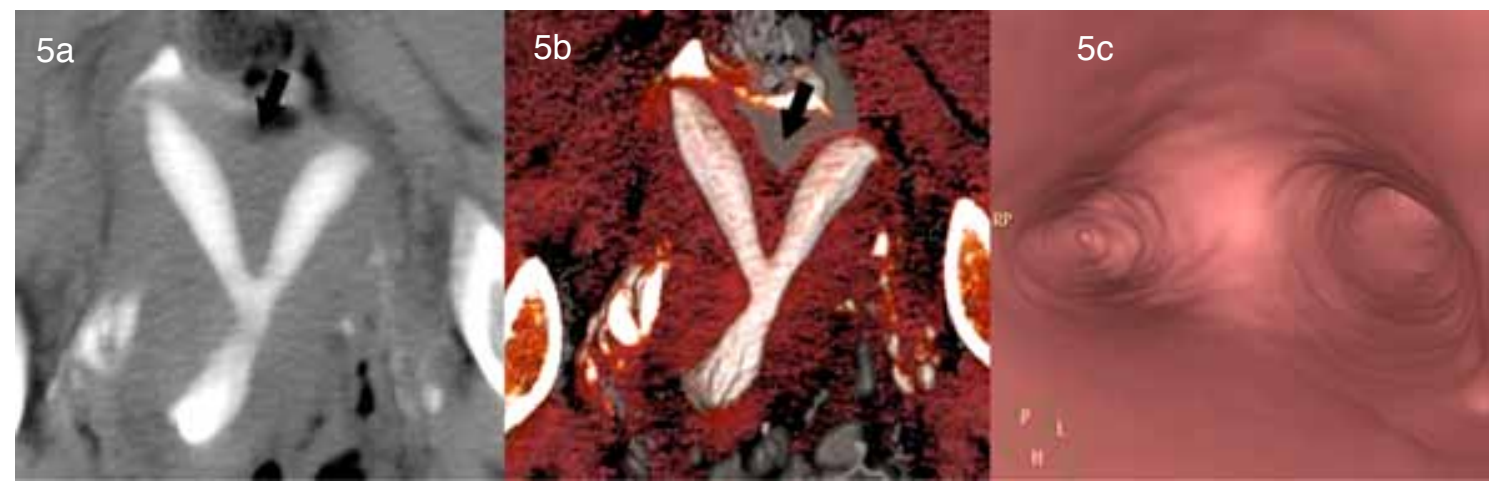

Figura 5. Útero bicorne. A. Reconstrucción coronal en máxima intensidad de proyección. B. Reconstrucción coronal 3D volumen rendering. C. Endoscopía virtual. La flecha indica la morfología externa del útero. 


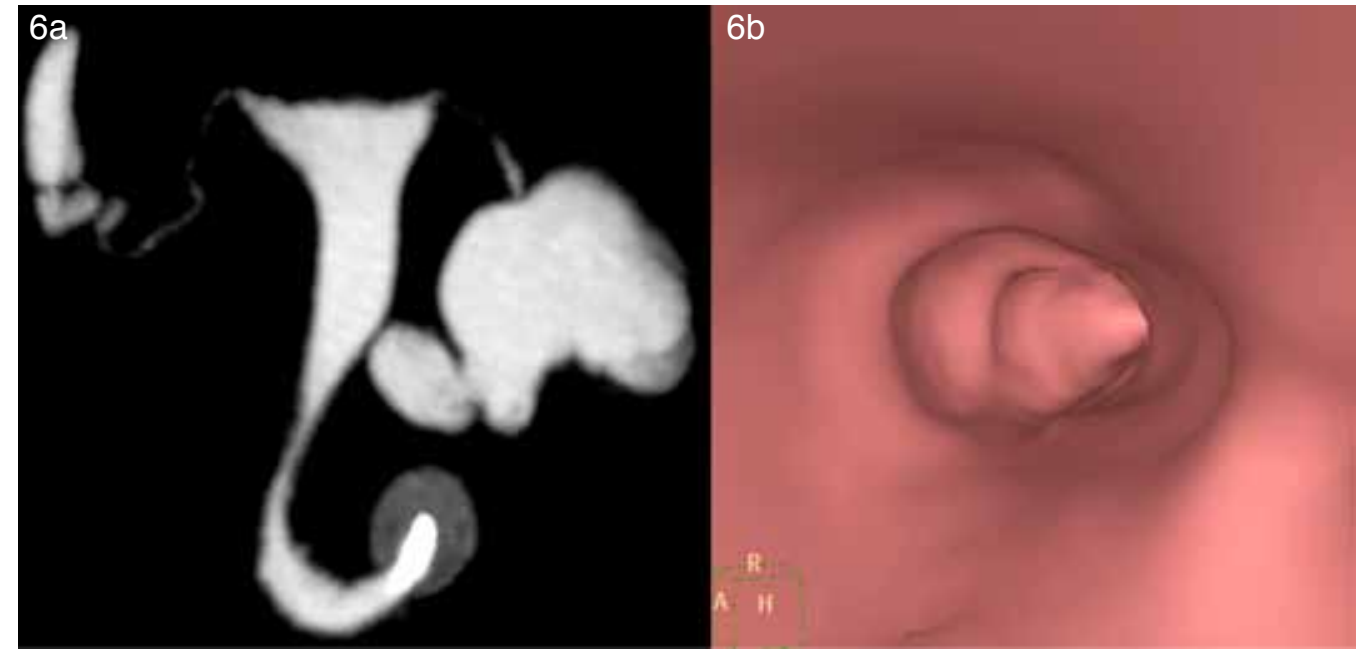

Figura 6. Hidrosalpinx izquierdo. A. Reconstrucción coronal en máxima intensidad de proyección que muestra una gran dilatación de la trompa uterina izquierda. B. Endoscopía virtual de la trompa dilatada.

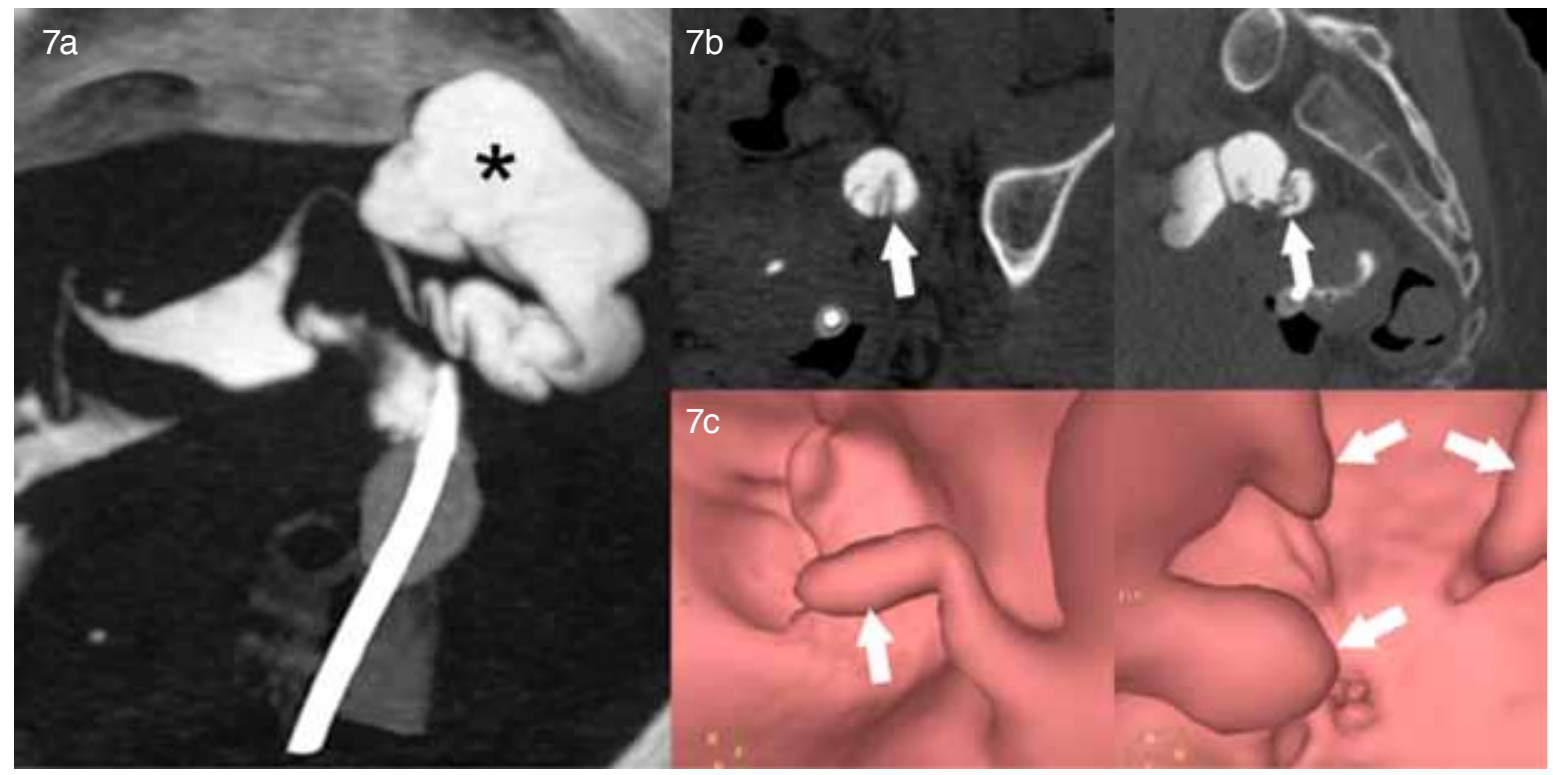

Figura 7. Hidrosalpinx izquierdo asociado a sinequias intratubarias. A. Reconstrucción coronal en máxima intensidad de proyección que muestra hidrosalpinx izquierdo (asterisco). B. Imágenes multiplanares de la trompa izquierda que muestran imágenes lineales endoluminales (flechas), correspondientes a sinequias. C. Endoscopía virtual de la trompa dilatada confirma los hallazgos endoluminales.

\section{Discusión}

La HSG-V es un método diagnóstico descrito recientemente, mínimamente invasivo. Nuestro grupo de trabajo ha comunicado resultados preliminares mostrando una buena performance del procedimiento para la evaluación del cérvix, cavidad endometrial y pared uterina ${ }^{(2)}$. El presente trabajo evalúa los resultados hallados en 2.500 estudios realizados con un tomógrafo de 64 filas de detectores y analiza otros aspectos relacionados. En el grupo con infertilidad secundaria que presentó un mayor número de abortos previos, fue muy importante la posibilidad de visualizar la cavidad uterina para el hallazgo de sinequias, así como la detección de anomalías congénitas y miomas.

La visualización de las imágenes axiales permite una evaluación integral de los órganos reproductores femeninos, facilitando la identificación de la patología miometrial (engrosamientos parietales, adenomiosis, miomas subserosos o intramurales), patología luminal (pólipos endometriales, sinequias, miomas submucosos) y también la evaluación de patología anexial $u$ otras patologías pelvianas. Las reconstrucciones MPR, combinadas con las imágenes en MIP, reconstrucciones 3D volume rendering y vistas endoscópicas, brindan una exacta caracterización de los diferentes tipos de anomalías del tracto re- 
productor femenino. Las reconstrucciones MPR son extremadamente útiles para una mejor delimitación y extensión de la patología, y para una medición exacta de las lesiones. Las reconstrucciones MIP brindan una visualización similar a la HSG así como una mejor determinación de la patología tubaria. Las imágenes 3D volume rendering ilustran la relación de los órganos reproductores femeninos con el resto de las estructuras pelvianas. Permiten además la remoción de las diferentes estructuras, utilizando una herramienta de sustracción por planos, de manera que el cérvix, útero y trompas de Falopio pueden ser rotados en diferentes orientaciones. El último paso en el análisis es la endoscopía virtual, que consiste en la vista endoluminal del cérvix, útero e incluso trompas de Falopio, demostrando la presencia de lesiones endocavitarias. Este algoritmo ofrece la posibilidad de crear imágenes similares a las obtenidas por histeroscopía, otorgando una adecuada delineación de las lesiones endocavitarias.

En relación al disconfort de las pacientes, esta técnica ha sido adecuadamente aceptada por la mayoría de las pacientes. Una ventaja importante en el procedimiento es la posibilidad de realizarlo sin la utilización de pinza erina ni otro tipo de sujeción ni tracción del cuello uterino.

Todos los estudios de HSG-V se realizaron usando una modulación automática del tubo de rayos $X$ durante el tiempo de escaneo, con el objetivo de reducir la dosis de radiación. En nuestra serie, la dosis efectiva de radiación promedio fue de 0.94 +/- $0.1 \mathrm{mSv}$.
En conclusión, la HSG-Virtual realizada con tomógrafos de 64 filas de detectores, es un método confiable, que permitió una adecuada evaluación de los órganos genitales internos, brindando información diagnóstica útil en infertilidad y otros desórdenes ginecológicos. Es una técnica poco dolorosa, bien tolerada por las pacientes y con baja dosis de radiación. Estas ventajas ubican a esta modalidad diagnóstica como una alternativa válida en el algoritmo de estudio de las pacientes con infertilidad.

\section{Bibliografía}

1. Rydberg J, Liang Y, Teague SD. Fundamentals of multichannel CT. Semin Musculoskelet Radiol 2004; 8: 137-146.

2. Carrascosa P, Baronio M, Capuñay C, López EM, Sueldo C, Papier S. Clinical use of 64-row multislice computed tomography hysterosalpingography in the evaluation of female factor infertility. Fertil Steril 2008; 90(5): 1953-1958.

3. Carrascosa P, Capuñay C, Mariano B, López EM, Jorge $\mathrm{C}$, Borghi M, et al. Virtual hysteroscopy by multidetector computed tomography. Abdom Imaging 2008; 33(4): 381-387.

4. Carrascosa P, Capuñay C, Baronio M, Martín López $\mathrm{E}$, Vallejos J, Borghi M, et al. 64-Row multidetector CT virtual hysterosalpingography. Abdom Imaging 2009; 34(1): 121-133.

5. Carrascosa P, Baronio M, Capuñay C, López EM, Vallejos J, Borghi M, et al. Multidetector computed tomography virtual hysterosalpingography in the investigation of the uterus and fallopian tubes. Eur J Radiol 2008; 67(3): 531-535. 